\title{
Thermodynamic functions of quantum electron gas in strongly anisotropic materials
}

\author{
C.C.Tovstyuk \\ Lviv Polytechnic National University, 12 S.Bandery Str., \\ 790013 Lviv, Ukraine
}

Received July 7, 2015

\begin{abstract}
In this paper we investigate thermodynamic functions of quantum electron gas in strongly anisotropic materials and compare them with the same functions obtained for isotropic material with quadratic dependence of energy on momentum. Our results are in a good agreement with theoretical conclusions of other authors and they help to explain a number of known experimental data obtained for these materials.

Keywords: strongly anisotropic, layered materials, thermodynamic functions, entropy, heat capacity.
\end{abstract}

Исследуются термодинамические функции квантового газа электронов в сильно анизотропных материалах, которые сравниваются с такими же функциями, полученными для изотропных материалов с квадратичной зависимостью энергии от импульса. Полученные результаты согласуются с теоретическими выводами других авторов и позволяют объяснить ряд известных экспериментальных данных, полученных для таких материалов.

Термодинамічні функції квантового електронного газу у сильно анізотропних матеріалах. К.К.Товстюк.

Досліджено термодинамічні функції квантового електронного газу у сильно анізотропних матеріаліх і зіставлено їх із такими ж функціями, отриманими для слабо ізотропного матеріалу із квадратичною залежністю енергії від імпульсу. Отримані результати узгоджуються із теоретичними висновками інших авторів та дозволяють пояснити низку відомих експериментальних даних, отриманих у таких матеріалах.

\section{Introduction}

Rapid progress of materials science inherent in this century forced researchers to engineering and developing the new nanomaterials. For such purposes the intercalation method (introduction of atoms and ions into Van der Waals gap of layered materials) seems to be very promising. Intercalation as nanotechnology instrument in various spheres of the modern science and technology is hard to overestimate. Thermodynamic (TD) investigations of the intercalation processes were carried out in [1-4]. But to understand the effects which take place after intercalation we need to know the TD functions and their peculiarities for electron gas in initial material ("host"). Here we cannot use the classical expressions because these materials are close to two dimensional structures. They consist of layers where structure units are binding with covalent and ion-covalent bounds and Van-derWaals binding between the layers [5, 6]. Layered materials $\mathrm{YTe}_{3}, \mathrm{LaTe}_{3}, \mathrm{CeTe}_{3}$ [7], InSe [8], as well as a number of organic conductors [9] belong to such structures.

Electron band calculations for organic conductors indicated the coexistence of equipotential surfaces in the form of corrugated cylinders and corrugated sheets [10]. In $\mathrm{YTe}_{3}, \mathrm{LaTe}_{3}, \mathrm{CeTe}_{3}$ equipotential energy surface, calculated from the first principles [11] contains weak dispersion along one di- 
rection. Two-dimensional metallic conductivity at the low temperatures in InSe was experimentally confirmed by quantum oscillations of magnetoresistance in [8].

Thermodynamic functions of the electron gas obtained in [12] revealed some differences from such functions in isotropic crystals, even for quasi-classical electron gas. Quasi-classical approximation is not applicable to materials with a high concentration of carriers and low temperatures. This case we consider in this paper.

\section{Approximations and expressions}

The layered crystals contain atoms of two or more layers in their unit cell with weak Van-der-Waals binding, which introduce, according to [13], only $2-3 \%$ of the whole boundary forces between the structure units. To consider this feature authors of [14-16] developed the expression for energy as quasimomentum function for strongly anisotropic materials (SAM), which is:

$$
\begin{gathered}
E(\mathbf{k})=\alpha k_{\perp}^{2}+\gamma(1-\cos z), \\
\alpha=\frac{\hbar^{2}}{2 m_{t}}, \quad \gamma=\frac{\hbar^{2} \pi^{2}}{4 m_{\|} d^{2}},
\end{gathered}
$$

where $m_{t}$ is effective mass of current carrier in the plane of the layers, $\gamma$ is halfwidth of the conduction band in the [001] direction, $z=k_{z} d, d$ is lattice translation this direction. Expression (1) determines the coexistence of open and closed equipotential surfaces for three- and two-dimensional electron gas. We compare the thermodynamic functions (TDF) for SAM with (1) and materials, in which energy depends on square momentum (PM). The TDF expressions for PM are known for example from [17]. For both examined cases we used the same parameters (effective mass and lattice translation) for GaSe from [18]. We found the density of states at the equipotential surface using the inversion theorem for the partition function [19]. The partition function for SAM was obtained in [20] and looks like:

$$
\begin{aligned}
& Z(\theta)=A \theta \exp (-\delta\} I_{0}(\delta), \\
& A=\frac{4 V L_{z} m_{t}}{h^{2} d^{2}}, \delta=\gamma / \theta,
\end{aligned}
$$

where $I_{0}(\delta)$ is the modified Bessel function. The resulting density of states (DS) coincides with the expressions obtained in [21].

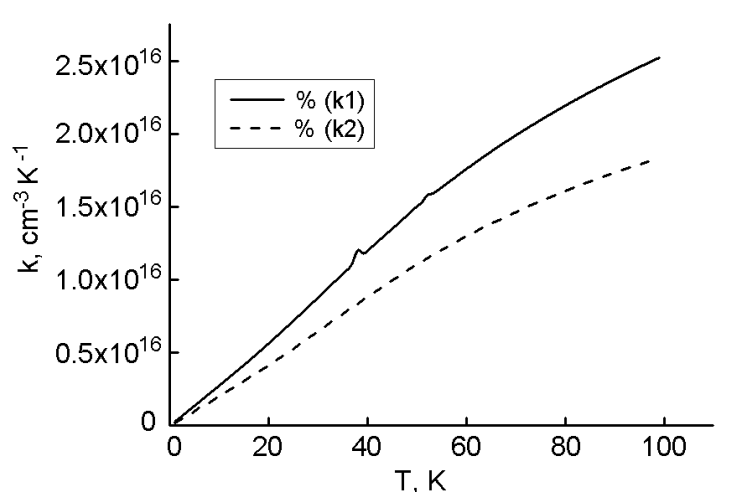

Fig. 1. Rate of concentration change with temperature for $\mathrm{SAM}(k 1)$ and $\mathrm{PM}(k 2)$.

Comparing the DS obtained for SAM with the DS for PM, we can see that:

- DS in SAM is substantially greater than the DS in PM for the small energies (low frequencies);

- DS in SAM is constant and significantly less than DS in PM (which is increasing with energy as $\sqrt{\varepsilon}$ ) for the high energies (frequencies).

The following calculations were carried out for expressions [22]:

$$
\begin{gathered}
N=\int_{0}^{\infty} g(\varepsilon) f_{F D}(\varepsilon) d \varepsilon, \\
E=\int_{0}^{\infty} \varepsilon g(\varepsilon) f_{F D}(\varepsilon) d \varepsilon, \\
-\Omega=\int_{0}^{\infty} \ln \left(1+\exp \left\{\frac{\mu-\varepsilon}{\theta}\right\}\right) g(\varepsilon) d \varepsilon .
\end{gathered}
$$

Heat capacity and entropy were calculated as derivative from energy and thermodynamic potential in temperature:

$$
\begin{gathered}
S(T)=k\left\{\int_{0}^{\infty} g(\varepsilon) \ln \left(1+e^{\frac{\mu-\varepsilon}{\theta}}\right) d \varepsilon-\right. \\
\left.-\int_{0}^{\infty} g(\varepsilon) f_{F D}(\varepsilon)\left(\frac{\mu-\varepsilon}{\theta}\right) d \varepsilon\right\}, \\
C(T)=k \int_{0}^{\infty} \varepsilon g(\varepsilon) f_{F D}(\varepsilon)\left(1-f_{F D}(\varepsilon)\right)\left(\frac{\varepsilon-\mu}{\theta^{2}}\right) d \varepsilon .
\end{gathered}
$$

Calculations were performed for the electron gas in $1 \mathrm{~cm}^{3}$ sample of $\beta$-GaSe, which is $0.034 \mathrm{~mol}$. 

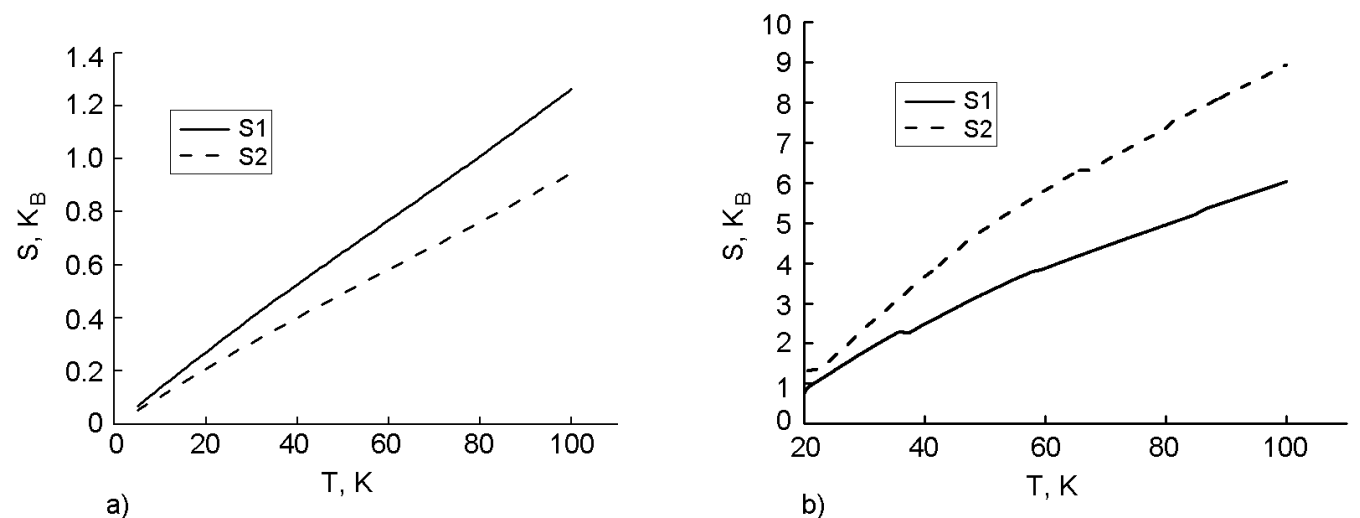

Fig. 2. Electron gas entropy for $\operatorname{SAM}\left(S_{1}\right)$ and $\mathrm{PM}\left(S_{2}\right)$ for a) $E_{F}=0.014 \mathrm{eV}$ (closed); b) $E_{F}=1 \mathrm{eV}$ (open equipotential surfaces).
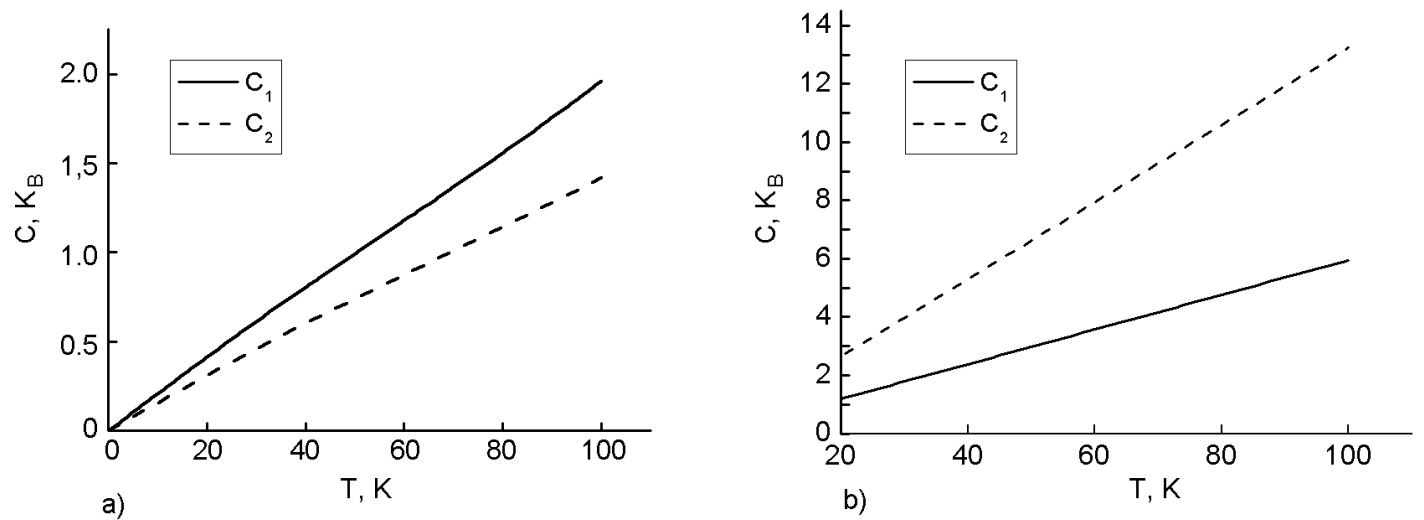

Fig. 3. Electron gas heat capacity for $\operatorname{SAM}\left(C_{1}\right)$ and $\mathrm{PM}\left(C_{2}\right)$ for a) $E_{F}=0.014 \mathrm{eV}$ (closed); b) $E_{F}=1 \mathrm{eV}$ (open equipotential surfaces).

\section{Results and discussions}

Data of the concentration of current carriers obtained from (3) shows that for the low temperatures in SAM number of electrons increases with temperature faster than in PM (Fig. 1, where $k=d n / d T$ ). Number of experimental works researching resistivity of GaSe at the low temperatures $[23,24]$ confirmed with this result. It is used in [25] for creation of the temperature sensor based on GaSe.

Heat capacity, received from (4), is a linear function of temperature with the inclination angle tangent of $0.053 \mathrm{~mJ} /\left(\mathrm{mol} \cdot \mathrm{K}^{2}\right)$.

Entropy and heat capacity are well interpolated by linear dependence on temperature with different tangent angles, depending on the Fermi energy. Fig. 2 and Fig. 3 show the dependences of entropy and heat capacity on the temperature for the Fermi energy of (a) $0.014 \mathrm{eV}$ and (b) $1 \mathrm{eV}$. As we see from Fig. 2, 3a), at the low Fermi energies (FE) the entropy and heat capacity of the electron gas SAM increases faster with temperature than for the PM. For large values of FE (Fig. 2, 3b) - on the contrary. These contradictory dependings we explain by the Lifshitz's phase transition - the transition from closed to open equipotential surfaces [26]. To better analyze the impact of this transition on the electron gas, we investigated thermodynamic functions dependence on the Fermi level. In result we did not note that any peculiarity in the thermodynamic potential and internal energy temperature dependences. Their absolute values grow with the temperature. However, the specific heat and entropy depending on the Fermi energy include the particular points for opening equipotential surfaces $\left(E_{F}=2 \gamma\right)$.

Thus, our study explains a number of experimental works, for example, [27], where the heat capacity for SAM is measured. Authors of [27] received special points and concluded the limitations of the Debye model for such materials. These conclusions agree with the results of [28-30] where phonon spectra of SAM was researched. The 

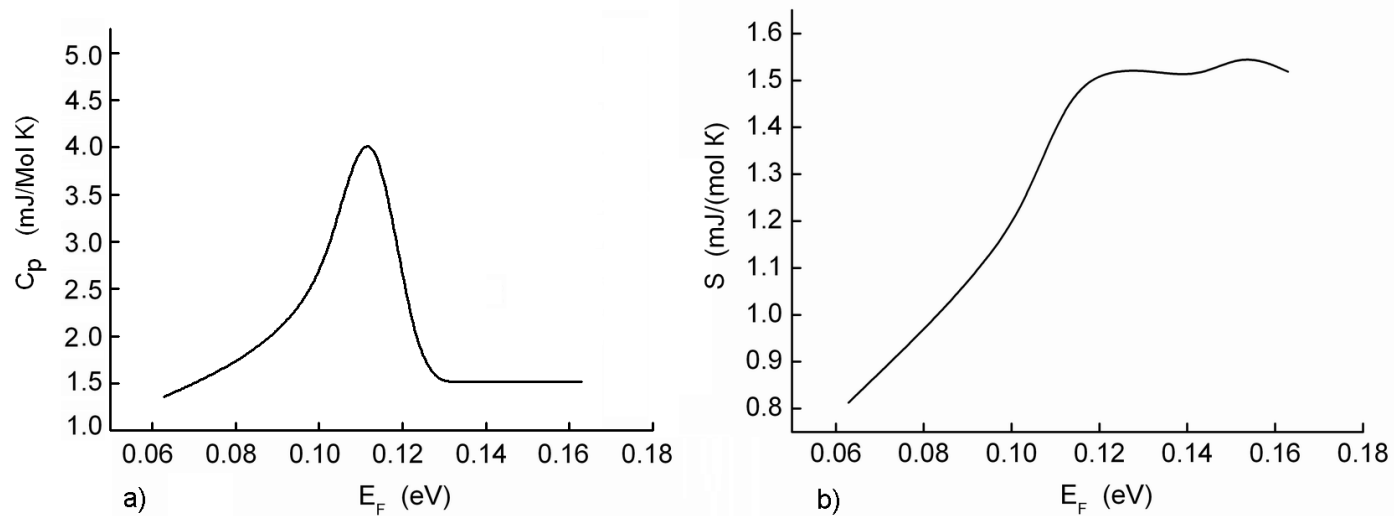

Fig. 4. Heat capacity (a) and entropy (b) of electron gas in SAM, depending on Fermi energy for $T=10 \mathrm{~K}$.

obtained dependence of the entropy on the Fermi energy agrees with the conclusions of [31] made for the entropy in two-dimensional gas.

\section{Conclusions}

The analysis of thermodynamic functions for electron gas in SAM and comparing with the same functions in PM showed. Rapid increase in the number of carriers with temperature at the low temperatures in the SAM. The rate of entropy and heat capacity increases have contradictory trends: dominates for SAM at closed equipotential surfaces (low energies, frequencies) and higher for the PM on open equipotential surfaces (high energies, frequencies). Thermodynamic potential and internal energy depend monotony on the Fermi energy (concentration of particles) both for the PM and SAM. Heat capacity and entropy dependences on the Fermi energy have special points in the transition from the closed to open equipotential surfaces.

Our results allowed to explain a number of the experimental data and agree with the conclusions of other authors for vibrational spectra and analytical investigations for the two-dimensional gas.

\section{References}

1. I.V.Myntianskyi, I.I.Grygorchak, Z.D.Kovaliuk, Solid State Phys., 28, 1263 (1986).

2. I.I.Grygorchak, Phys. Chem. Sol. State. 88, 3 (2002).

3. I.D.Kozmyk, Z.D.Kovaliuk, I.I.Grygorchak, B.P.Bahmatiuk, in: Proc. of the Acad. Sci. Soviet Union, Inorganic Materials, v.23 (1987), p.754.

4. I.I.Grygorchak, in: Material Science of Low Energy Gap and Layered Semiconductors, Naukova Dumka, Kiev (1989) [in Russian].
5. D.I.Bletskan, V.V.Vakulchak, K.E.Glukhov, Appl. Phys. A, 117, 1499 (2014).

6. D.I.Bletskan, V.V.Vakulchak, V.M.Kabatsii, Open J. Inorg. Non-metal. Mater., 5, 31 (2015).

7. I.R.Fisher, Phys.Rev.B, 73, 033101 (2006).

8. N.B.Brandt, V.A.Kulbachynskyy, Z.D.Kovaliuk, G.V.Lashkarev, Phys.Techn.Semicond., 21, 1230 (1987).

9. N.Ru, O.V.Kirichenko, V.G.Peschanskiy, R.A.Khasan, J.Exper.Theor.Phys., 132, 183 (2007).

10. J.Laverock, S.B.Dugdale, Zs.Major et al., Phys.Rev. B, 71, 085114 (2005).

11. M.V.Karcovnik, V.G.Peschanskiy, Phys. Low Temp., 31, 249 (2005) .

12. C.C.Tovstyuk, Chem.Met.Alloys, 4, 58 (2011).

13. A.A.Balchin, Growth and Crystal Characteristics of Dichalcogenides Having Layer Structure, D.Reidel Publ.Co., Dortrecht-Holland (1976).

14. R.F.Fivas, E.Mosser, Phys.Rev., 136, 833 (1964).

15. R.F.Fivas, J.Phys.Chem.Solids, 28, 839 (1967).

16. R.C.Fivaz, Ph.E.Schmidt, in: Optical and Electrical Properties of Material with Layer Structures, D.Reidel Publ.Co., Dortrecht-Holland (1976).

17. L.D.Landau, E.M.Lifshitz, Statistical Physics, Nauka, Moscow (1964) [in Russian].

18. O.Madelung, M.Schulz, H.Weiss (Eds.), Halbleiter. Landolt-Bornstein III/17, SpringerVerlag, Berlin (1983).

19. A.G.Samoilovych, Thermodynamics and Statistical Physics, Publishing House of Technical and Theoretical Literature, Moscow (1999) [in Russian].

20. C.C.Tovstyuk, Mol.Phys.Reports, 23, 197 (1999).

21. N.K.Tovstyuk, Phys. of Low Temperatures, 30, 672 (2004)

22. C.Kittel, Basic Statistical Physics, Publ. House for Foreign Literature, Moscow (1960) [in Russian]. 
23. A.M.Pashayev, A.R.Gadjiyev, T.B.Tagiyev, T.M.Abbasova, Semicond. Phys., Quant. Electron. Optoelectron., 4, 287 (2001).

24. A.Geydarov, J. Inorgan. Chem., 52, 1618 (2007).

25. V.O.Khandozhko, Technol. Audit and Prod. Res., 5, 13 (2013).

26. I.M.Lifshitz, Zh. Eksper. Teor. Fiz., 22, 471 (1952).

27. J.E.Callanan, R.D.Weir, E.F.Westrum, Pure Appl.Chem., 69(11), 2289 (1997).
28. I.M.Gelflart, Ye.S.Syrkin, Phys. Low Temperatures. 4, 672 (1978).

29. Ye.S.Syrkin, I.M.Gelflart, Phys. Low Temperatures, 5, 181 (1979).

30. I.A. Gospodarev, V.I.Grishiayev, S.B.Feodosyev et al., Phys. Low Temperatures, 34829 (2008).

31. V.M.Gohfeld, Phys.Low Temperatures, 31, 769 (2005). 